\title{
PERFIL CLÍNICO E TERAPÊUTICO DA NEURITE CAUSADA PELA HANSENÍASE
}

\author{
CLINICAL AND THERAPEUTIC PROFILE OF NEURITIS CAUSED BY \\ LEPROSY
}

Gabriel Martinez Andreola1; Vanessa Cristhine Dallolmo Ottoboni2; Maira Mitsue Mukai3

\section{RESUMO}

OBJETIVO: Avaliar as características clínicas e terapêuticas dos pacientes com neurite no Ambulatório de Hanseníase em Hospital Universitário entre janeiro de 2005 e dezembro de 2013. MÉTODOS: Estudo observacional, retrospectivo, baseado na revisão de prontuários. Foram analisados 108 prontuários e a análise estatística foi feita por médias e medianas (Microsoft Exce/ ${ }^{\circledR}$ ). RESULTADOS: A média de idade foi de 49,3 anos. Aproximadamente $67 \%$ da população eram homens e a maioria dos pacientes é procedente do Paraná, sendo que boa parte pertencente à Curitiba e região metropolitana. As formas multibacilares prevaleceram, com a forma virchowiana (40,2\%), seguida pela dimorfa (27,1\%). Dos pacientes, $34 \%$ apresentaram neurite. 0 espessamento neural foi detectado em mais da metade dos pacientes e quase $50 \%$ dos pacientes desenvolveram reações hansênicas, mais comuns nos multibacilares. Cerca de $75 \%$ dos doentes foram submetidos a avaliação funcional inicial e 45,6\% destes apresentavam algum grau de incapacidade ao diagnóstico. Dos 37 pacientes com neurite em uso de poliquimioterapia específica associada ao tratamento da neurite, apenas 4 melhoram o grau funcional, enquanto 2 pacientes tiveram piora e em 18 indivíduos houve apenas a estabilização do quadro clínico. CONCLUSÃo: Foi observado predomínio das formas multibacilares e a presença significativa da neurite nas reações hansênicas. Houve estabilização das incapacidades funcionais com as terapêuticas atuais utilizadas no Serviço.

Descritores: Hanseníase, Neurite, Infecção.

\section{ABSTRACT}

OBJECTIVE: Evaluate the clinical and therapeutic characteristics of patients with neuritis in Leprosy Clinic at University Hospital between January 2005 and December 2013. METHODS: Observational and retrospective study based on medical records review. About 108 medical records were analyzed and the statistical analysis was performed by means and medians (Microsoft Excel $\left.{ }^{\circledR}\right)$. RESULTS: The mean age was 49.3 years, approximately $67 \%$ of the population were men, the majority of patients are coming from Paraná, with much of belonging to the metropolitan area of Curitiba. The multibacillary forms prevailed, with lepromatous (40.2\%), followed by borderline (27.1\%). Thirty four percent of patients had neuritis. The nerve enlargement was detected in more than half of patients and almost $50 \%$ of patients developed leprosy reactions, most common in multibacillary. Approximately $75 \%$ of patients underwent initial functional assessment and $45.6 \%$ of them had some degree of disability at diagnosis. Few patients confirmed the diagnosis by biopsy or nerve electromyography. Thirty seven patients with neuritis using specific multidrug therapy associated with the treatment of neuritis, only 4 upgrade functional class, while 2 patients worsened and 18 patients there was only the stabilization of the clinical status. CONCLUSION: It was observed a predominance of multibacillary and the significant presence of neuritis in leprosy reactions. There was stabilization of functional disabilities with current therapies used in the Service.

Keywords: Leprosy, Neuritis, Infection.

\footnotetext{
1 - Acadêmico do curso de Medicina, Universidade Federal do Paraná, Curitiba, Paraná, Brasil.

2 - Médica Dermatologista, Universidade Federal do Paraná Curitiba, Paraná, Brasil.

3- Professora adjunta da disciplina de Dermatologia, Departamento de Clínica Médica, Universidade Federal do Paraná, Curitiba, Paraná, Brasil.
}

Contato do Autor / Mail to:

Gabriel Martinez Andreola

Rua Ubaldino do Amaral, 360, apto. 05, Alto da Glória, Curitiba - PR, 80060-195.. 


\section{INTRODUÇÃO}

A Hanseníase, antigamente conhecida como Lepra ou Mal de Hansen, é uma doença infectocontagiosa considerada um problema de saúde pública em diversos países em desenvolvimento. Seu agente etiológico é o Mycobacterium leprae ${ }^{1}$ um bacilo ácido álcool resistente (BAAR)1,2. O Brasil apresentou uma prevalência de 1,56 para 10.000 habitantes em 2010, ocupando o segundo lugar mundial em número de casos $2,3,4$.

A resposta imune do hospedeiro está associada ao desenvolvimento de formas clínicas distintas da doença, denominadas: Indeterminada, Tuberculóide ${ }^{1,5}$, Virchowiana6,7 e Dimorfa1. A forma Indeterminada é considerada como a forma inicial da doença, que pode evoluir para qualquer uma das outras formas. Já a Dimorfa abrange aspectos tanto da Tuberculóide, quanto da Virchowiana1. Esta classificação é realizada de acordo com aspectos morfológicos das lesões e a presença ou não de bacilos no exame de baciloscopia, o qual consiste na análise da linfa corada por Ziehl-Neelsen obtida através de um raspado dérmico, comumente dos lóbulos das orelhas ${ }^{1,5-7}$

Uma outra forma mais rara de apresentação da Hanseníase é denominada forma Neural Pura. Esta é caracterizada apenas por manifestações neurológicas, principalmente em nervos mistos, com alterações sensitivas, motoras e/ou autonômicas nas extremidades, sem evidência de lesões cutâneas ${ }^{6}$. 0 exame eletroneuromiográfico tem maior especificidade e sensibilidade para investigar a localização e distribuição do comprometimento8, mas seu diagnóstico definitivo é estabelecido pela biópsia neural 9 . Os principais troncos nervosos acometidos na hanseníase são radial, ulnar, tibial posterior, fibular comum, mediano, cervical e auricular ${ }^{1,10}$.

As alterações nos nervos, por ação direta do bacilo ou reação imunológica do próprio organismo, são responsáveis por sequelas permanentes, comprometendo a qualidade de vida e a capacidade laborativa ${ }^{1}$. Para a avaliação das sequelas é importante o estadiamento através da avaliação funcional: no Grau 0 não se apresenta nenhuma alteração relacionada a hanseníase; no Grau 1 há uma alteração de sensibilidade em olhos e dimídios; e no Grau 2 já existem alterações oftalmológicas mais graves, lesões tróficas nos membros e deformidades ${ }^{11}$.

O Mal de Hansen tem seu diagnóstico confirmado através da presença de lesões de pele com alterações da sensibilidade, acometimento de nervos com espessamento neural e/ou baciloscopia positiva12.

O tratamento da Hanseníase é realizado com poliquimioterapia de acordo com a classificação da doença. O esquema paucibacilar é composto por Rifampicina e Dapsona, pode durar de 6 a 9 meses e é indicado para as formas Indeterminada, Tuberculóide 5 e na forma Neural Pura com acometimento de um único nervo 9 . O esquema multibacilar é composto por Rifampicina, Clofazimina e Dapsona, podendo durar de 12 a 24 meses e é utilizado para as formas Dimorfa, Virchowiana ${ }^{7}$ e Neural Pura com mais de um nervo acometido13. Existem outros esquemas alternativos e individualizados ${ }^{5}$.

Em média, após cinco anos do início do tratamento podem ocorrer as reações hansênicas, que são episódios inflamatórios agudos que se sobrepõem à evolução crônica e insidiosa da doença. É preciso uma intervenção imediata, pois podem acarretar perda funcional de nervos periféricos e agravamentos das incapacidades 1,14 . Existem três formas clínicas de episódios reacionais: Reação Reversa (RR) ou tipo 1; Eritema Nodoso Hansênico $(E N H)$ ou reação tipo 2; e Neurite Isolada (NI) ${ }^{14,15}$.

A reação hansênica tipo 1 é uma reação de hipersensibilidade tipo IV de Gell e Coombs, ja reação tipo 2, uma hipersensibilidade tipo III. Apresentam novas ou reativações das lesões e nódulos eritematosos, respectivamente, além de sintomas sistêmicos, como febre, astenia, mialgia, dor articular, que são mais comuns no $\mathrm{ENH}^{14}$. A neurite, que pode estar associada as outras reações ou se apresentar de forma isolada, é caracterizada pela presença de disestesia, parestesia e/ou alterações motoras devido, principalmente, à compressão nervosa pelo edema do processo inflamatório, sendo que a NI não apresenta alterações cutâneas e sistêmicas características das outras reações ${ }^{14}$. A neurite pode se apresentar ainda de forma silenciosa, com atrofia progressiva e sem sintomas ${ }^{14}$.

0 tratamento das reações hansênicas é baseado no tipo e gravidade da reação, podendo ser usados antiinflamatórios não esteroidais, Talidomida, Clofazimina, Pentoxifilina e corticóides. Nos casos de neurite, o tratamento pode variar de repouso absoluto à descompressão cirúrgica (neurólise). Descartando-se a presença de quadro reacional, a dor neuropática, caracterizada por doença ou dano no sistema nervoso central ou periférico ${ }^{16}$, pode ser tratada com antidepressivos tricíclicos, neurolépticos e/ou anticonvulsivantes ${ }^{17}$.

0 objetivo deste trabalho é avaliar as características clínicas e terapêuticas dos pacientes com neurite no Ambulatório de Hanseníase em Hospital Universitário entre janeiro de 2005 e dezembro de 2013.

\section{MÉTODOS}

0 presente estudo caracteriza-se como observacional e retrospectivo. Os critérios de inclusão compreenderam pacientes maiores de 18 anos, com prontuários disponíveis, diagnosticados com hanseníase e tratados no Ambulatório de Hanseníase do Serviço de Dermatologia do Hospital de Clínicas da Universidade Federal do Paraná.

Foram revisados 108 prontuários no período de Janeiro de 2005 a Dezembro de 2013. O trabalho foi aprovado pelo Comitê de Ética e Pesquisa da instituição com o parecer n645.856/2014. 
Os dados pesquisados compreenderam itens como a idade, forma clínica da doença, tratamentos, tempo de tratamento, sequelas, avaliação funcional inicial e final e presença de reações.

Os critérios clínicos definidores de neurite foram a presença de dor neural com ou sem espessamento neural, alteração no exame neurológico, como diminuição da sensibilidade ou da força motora no trajeto do nervo, ou piora do grau de incapacidade na avaliação dermatoneurológica realizada pela terapeuta ocupacional do Serviço em relação ao exame anterior.

A análise estatística foi baseada em médias e desvio-padrão utilizando a planilha eletrônica (Microsoft Excel ${ }^{\circledR}$ ). Considerando o tipo de hipótese casuística não houve variáveis dependentes ou independentes.

\section{RESULTADOS}

Verificamos que $67,5 \%$ dos pacientes eram homens e a média de idade foi de 49,4 anos ( $\pm 15,9$ anos). Aproximadamente, $97 \%$ da amostra procederam do Paraná. A forma clínica da Hanseníase mais prevalente foi a Virchowiana com $40,2 \%$ da amostra, seguida pela Dimorfa $(27,1 \%)$, Tuberculóide $(19,7 \%)$, Indeterminada $(8,4 \%)$ e Neural Pura (4,6\%).

No tratamento, o número de cartelas utilizadas foram em média $8,9( \pm 5,6)$. As reações hansênicas estavam presentes em $62,9 \%$ dos indivíduos, sendo que a neurite representou $54,4 \%$ dos doentes. Cerca de $60 \%$ dos indivíduos apresentavam espessamento neural e $28,70 \%$ possuíam alguma sequela, das quais $70,9 \%$ eram neurológicas.

Os dados da avaliação funcional geral dos pacientes bem como sua evolução durante o tratamento estão demonstrados na tabela 1 e as alterações neurológicas entre a primeira e a última avaliação, na tabela 2. Dos pacientes com alterações neurológicas, durante a avaliação inicial, 54,05\% apresentavam um território nervoso afetado e 45,95\% manifestaram mononeuropatia múltipla. Na avaliação final, foi observado que $42,85 \%$ apresentavam apenas um tronco nervoso afetado e o restante em vários territórios. Apenas 4,6\% dos pacientes fizeram biópsia de tronco nervoso, todos com resultados positivos, e apenas $1,8 \%$ fizeram eletroneuromiografia.

A forma neural pura representou 4,6\% da amostra, todos do sexo masculino, com média de idade de 45,2 anos ( $\pm 16,6$ anos). Estes realizaram tratamento com esquema multibacilar, com uma média de 10,5 cartelas $( \pm 5,2$ cartelas $)$ e por aproximadamente 11 meses $( \pm 7,5$ meses). 0 perfil das alterações funcionais iniciais destes doentes se distribuiu em Grau 1 (20\%) e Grau 2 (60\%). No exame final, $60 \%$ foram classificados como Grau 2. 0 espessamento neural foi descrito em $60 \%$ destes casos. Mostrou-se que $80 \%$ destes indivíduos apresentavam sequelas neurológicas. Apenas duas pessoas realizaram biópsia de nervos e uma realizou a eletroneuromiografia.
Tabela 1: Avaliação do grau funcional e evolução no seguimento da amostra (\%).

\begin{tabular}{|l|c|c|c|}
\hline Avaliação & Grau 0 & Grau 1 & Grau 2 \\
\hline Inicial & 40,7 & 20,3 & 13,8 \\
\hline Final & 46,8 & 17,8 & 10,2 \\
\hline Evolução & $\begin{array}{c}\text { Grau } \\
\text { mantido }\end{array}$ & Melhora & Piora \\
\hline & 73,8 & 15,4 & 10,8 \\
\hline
\end{tabular}

Grau 0 sem alterações; Grau 1 alterações leves/Moderadas; Grau 2 alterações graves;

Aproximadamente de $34 \%$ dos pacientes estudados apresentaram neurite, sendo que a média da persistência da reação foi de 15,2 meses ( $₫ 20,9$ meses). Oitenta e um por cento são homens, com uma média de idade de 49,9 anos ( $\pm 13,4$ anos). Cerca de $81 \%$ usavam o esquema multibacilar e o restante, o paucibacilar. 0 tempo de tratamento foi de 11,2 meses ( $\pm 6,4$ meses), com um número médio de cartelas usadas de 10,5 ( $\pm 5,2$ cartelas). Para o tratamento da neurite, usou-se prednisona (86,48\%), talidomida (35,13\%), amitriptilina (40,54\%), gabapentina $(8,1 \%)$, carbamazepina, pentoxifilina e/ou anti-inflamatórios não esteroidais $(2,7 \%$ cada). 0 quadro de alterações funcionais iniciais da neurite $(78,3 \%$ dos pacientes com neurite) e a evolução da funcionalidade estão representados na Tabela 3 . Dos indivíduos com neurite, independente da forma clínica presente, 5,4\% fizeram biópsia de nervos e realizaram a eletroneuromiografia.

Tabela 2: Alterações sensitivas, motoras e tróficas durante avaliacções funcionais (N)

\begin{tabular}{|c|c|c|}
\hline & Avaliação inicial & Avaliação final \\
\hline $\begin{array}{l}\text { Sensitivas em } \\
\text { mãos }\end{array}$ & 13 & 6 \\
\hline $\begin{array}{l}\text { Sensitivas em } \\
\text { pés }\end{array}$ & 26 & 14 \\
\hline $\begin{array}{l}\text { Sensitivas em } \\
\text { olhos }\end{array}$ & 5 & 5 \\
\hline $\begin{array}{l}\text { Sensitivas em } \\
\text { outros locais }\end{array}$ & 6 & 1 \\
\hline $\begin{array}{l}\text { Motoras (mão } \\
\text { em garra) }\end{array}$ & 6 & 6 \\
\hline Tróficas & 2 & 3 \\
\hline
\end{tabular}

N: número absoluto 
Tabela 3: Avaliação e evolução funcional dos pacientes com neurite (\%)

\begin{tabular}{|c|c|c|c|}
\hline Avaliação & Grau 0 & Grau 1 & Grau 2 \\
\hline Inicial & 24,3 & 32,4 & 21,6 \\
\hline Final & 27,0 & 27,0 & 24,3 \\
\hline Evolução & $\begin{array}{c}\text { Grau } \\
\text { mantido }\end{array}$ & Melhora & Piora \\
\hline & 83,8 & 10,8 & 5,4 \\
\hline
\end{tabular}

Grau 0 sem alterações; Grau 1 alterações leves/Moderadas; Grau 2 alterações graves;

\section{DISCUSSÃO}

$\mathrm{Na}$ avaliação dos pacientes com hanseníase, observamos que mais da metade eram homens e a média de idade se aproximou de 50 anos. A maioria dos pacientes é procedente do Paraná, sendo que boa parte pertencente à Curitiba e região metropolitana. Assim como na literatura1,5-7, a principal forma da doença foi a virchowniana, seguida da dimorfa, tuberculóide, indeterminada e, em menor frequência, forma a neural pura. No tratamento da hanseníase, foram usadas uma média de 8,9 cartelas, enquanto os pacientes com a forma neural pura ou que possuíam neurite como manifestação usaram em torno de 10,5 cartelas, não encontramos este dado para comparação na literatura.

O espessamento neural e as alterações neurológicas, sem as lesões características de pele da hanseníase, caracterizam a forma neural pura ${ }^{9}$, em nosso estudo, 5 pacientes apresentavam esta forma, destes, dois realizaram biopsia de troncos nervosos e apenas um realizou a eletroneuromiografia, todos apresentando resultados positivos. Considerando as substanciais diferenças, estes dados corroboram com outros resultados, cuja suspeita da forma neural pura confirmouse pelo exame eletrofisiológico e pela biópsia18.

As reações hansênicas são as principais causas de lesões dos nervos e incapacidades provocadas pela hanseníase ${ }^{19}$. As reações estavam presentes em 62,9\% dos indivíduos, sendo que mais da metade apresentavam também a neurite no quadro clínico. Considerando a totalidade da amostra, 28,7\% apresentavam sequelas, das quais $70,9 \%$ eram neurológicas, incluindo o espessamento neural (60\%), tal realidade se aproxima dos valores encontrados na literatura, na qual $78,9 \%$ possuíam alguma alteração sensitivo-motora e $68,4 \%$ espessamento de algum tronco nervoso ${ }^{9}$.

A neurite estava presente em $34,25 \%$ do total da amostra, valor maior que o encontrado em outros trabalhos com doentes multibacilares $(7 \%)^{14}$. Dos afetados por neurite, $81,08 \%$ são homens, e não encontramos relatos referentes a este dado. A média de idade foi de 49,9 anos, semelhante a encontrada em outros levantamentos 20 . A maioria dos pacientes com neurite eram multibacilares, em uso de poliquimioterapia com três drogas.

A neurite foi tratada principalmente com prednisona (86,48\%), seguida pela talidomida, amitripilina e gabapentina. A prednisona foi uma droga utilizada com frequência, pois alivia a compressão das fibras pelo edema, contribuindo para a diminuição dos sintomas. As outras medicações também apresentam respaldo para o uso ${ }^{17}$. A média de tempo de persistência da reação foi de 15,2 meses e a duração do tratamento foi de quase um ano. De acordo com outros estudos, o período de tratamento de 4 a 9 meses de corticoterapia é eficiente para prevenção de reações duradouras ${ }^{21}$.

0 quadro de alterações funcionais iniciais nos doentes com neurite divide-se em graus. Dos pacientes que apresentavam dados completos, demostrou-se que o tratamento leva ao alívio dos sintomas e a uma estabilização das incapacidades funcionais, ou seja, a não piora da evolução das incapacidades, já que 48,6\% não mudaram de grau entre os exames, 10,8\% melhoraram e $5,4 \%$ apresentaram piora da funcionalidade. Estes dados são relativamente coerentes aos de outros estudos 22,23 , o que evidencia a gravidade em que a doença afeta os nervos periféricos. As principais alterações clínicas iniciais presentes foram disestesia, parestesia e/ou alterações motoras e estão descritas na tabela 2 . A redução da intensidade dos quadros relacionados à sensibilidade dos membros foram as mais evidentes, sugerindo um benefício do tratamento para a reação neural ${ }^{19,22}$.

0 baixo índice de realização de exames padrãoouro (biópsias de nervos e eletroneuromiografia) no presente estudo pode ser discordante com o manejo adequado daqueles que apresentam indicação para a sua realização, pois os mesmos são exames de significativo valor para a avaliação neurológica. Guardadas as proporções, estes resultados corroboram com o de outro estudo mais preciso, em que todos os indivíduos que tiveram indicação para a realização destes exames apresentaram algum acometimento neural comprovado devido a Hanseníase 8 .

\section{CONCLUSÃO}

Foi observado predomínio das formas multibacilares e a presença significativa da neurite nas reações hansênicas. Houve estabilização das incapacidades funcionais com as terapêuticas atuais utilizadas no Serviço.

\section{CONFLITOS DE INTERESSE}

Não houve conflitos de interesse. 


\section{REFERÊNCIAS}

1. Araújo MG. Hanseníase no Brasil. Soc Bras Med Tropical. 2003; 36: 373-82.

2. Sanches LAT, Pittner E, Sanches HF et Monteiro MC. Detecção de casos novos de hanseníase no município de Prudentópolis, PR: uma análise de 1998 a 2005. Soc Bras Med Tropical. 2007; 40: 54145

3. Longo JDM, Cunha RV. Perfil clínico-epidemiológico dos casos de hanseníase atendidos no hospital universitário em Campo Grande, Mato Grosso do Sul, de janeiro de 1994 a julho de 2005. Hansen Int. 2006; 31: 9-14.

4. Penna GO, Pinheiro AM, Nogueira LSC, Carvalho LR, Oliveira MBB, Carreiro VP. Clinical and epidemiological study of leprosy cases in the University Hospital of Brasília: 20 years - 1985 to 2005. Rev Soc Bras Med Tropical. 2008; 41: 575-80.

5. Hanseníase: atividades de Controle e Manual de Procedimentos. Ministério da Saúde.2004. Elaboração: área técnica de Dermatologia Sanitária, 2004. ISBN 85-334-0540-5.

6. Foss NT. Aspectos imunológicos da Hanseníase. Med Ribeirão Preto. 1997; 30: 335-39.

7. Mendonça VA, Costa RD, Melo GEBA, Antunes CM, Teixeira AL. Imunologia da Hanseníase. An Bras Dermatol. 2008; 83: 343-50.

8. Skacel M, Antunes SLG, Rodrigues MMJ, Nery JAC, Valentim VC, Morais RPB, et al. The diagnosis of leprosy among patients with symptoms pf peripheral neuropathy without cutaneous lesions. Arq Neuropsiquiatr. 2000; 58: 800-07.

9. Jardim MR, Chimeli L, Faria SC, Fernandes PV, Néri JAC, Sales AM, Sarno EM Antunes SLG. Cinical, Electroneuromyographic and morphological studies of pure neural leprosy in a Brazilian referral centre. Lepr Rev. 2004; 75: 242-253.

10. Lockwood DNJ, Suneetha S. Leprosy: too complex a disease for a simple elimination paradigm. Bull World Health Organization. 2005; 83: 230-35.

11. Ferreira TL, Alvarez RRA, Virmond MCL. Questionnaire on hand assessment in leprosy. Rev Saúde Pública 2012; 46: 435-45.

12. Brasil. Ministério da Saúde. Secretaria de Políticas de Saúde. Departamento de Atenção Básica. Guia para - Controle da hanseníase. Brasília: Ministério da Saúde, 2002.ISBN 85-334-0346-1.

13. Word Health Organization - WHO. Expert committee on leprosy: Technical report series. 1998; 874:1-43.

14. Nery JAC, Sales AM, Illarramendi X, Duppre NC, Jardim MR, Machado AM. Contribuição ao diagnóstico e manejo dos estados reacionais. Uma abordagem prática. An Bras Dermatol. 2006; 81: 367-75.

15. Cuevas J, Rodriguez-Peralto JL, Carrillo R, Contreras F. Erythema Nodosum Leprosum: reactional leprosy. Sem Cut Med and Surgery. 2007; 26: 126-30.

16. Haanpää M, Lockwood DNJ, Hietaharju A. Neuropathic pain. Lepr Rev. 2004; 75: 7-18.
17. Foss NT. Episódios reacionais na hanseníase. Medicina, Ribeirão Preto. 2003; 36: 453-59.

18. Garbino JA, Ura S, Belone AFF, Marciano, LHSC, Fleury RN. Aspectos clínicos e diagnósticos da hanseníase primariamente neural. Hansen Int. 2004; 29: 124-129.

19. Souza LWF. Reações hansênicas em pacientes alta por cura. Rev. Soc. Bras. Med. Trop. 2010; 43: 737 39.

20. Antonio JR, Soubhia RMC, Paschoa VD, Oliveira GB, Rossi NCP, Maciel MG, et al. Avaliação epidemiológica dos estados reacionais e incapacidades físicas em pacientes com hanseníase na cidade de São José do Rio Preto. Arq Ciênc Saúde. 2011; 18: 9-14.

21. Yawalkar SJ. Leprosy for medical practioners and paramedical workers. World Health Organization Novartis Foundation for Sustainable Development, Basle, Switzerland, Chapter. 2002; 12: 72-9.

22. Pimentel MIF, Nery JAC, Borges E, Rolo R, Sarno EN. Neurite silenciosa na hanseníase multibacilar avaliada através da evolução das incapacidades antes, durante e após a poliquimioterapia. An bras Dermatol. 2004; 79: 169-179.

23. Cardoso FM, De Freitas MR, Escada TM, Nevares MT, Nascimento OJ. Late onset neuropathy in leprosy patients released from treatment: not all due to reactions. Lepr Rev. 2013; 84: 128-135. 